\title{
ASSESSMENT OF SERUM LEVEL OF PROSTATE-SPECIFIC ANTIGEN ADJUSTED FOR THE TRANSITION ZONE VOLUME IN EARLY DETECTION OF PROSTATE CANCER
}

\author{
MARCOS D. FERREIRA, WALTER J. KOFF \\ Division of Urology, General Hospital of Porto Alegre, Federal University of Rio Grande do Sul, UFRGS, \\ Porto Alegre, Rio Grande do Sul, Brazil
}

\begin{abstract}
Objective: To determine the clinical usefulness of prostate-specific antigen (PSA) density in the transition zone (PSADTZ) for increasing the specificity in early detection of prostate cancer (PCa) and reducing unnecessary biopsies in males with PSA between 4.0 and $10 \mathrm{ng} / \mathrm{mL}$.

Materials and Methods: This cross-sectional study obtained PSADTZ measurements in 68 patients with PSA between 4.0 and $10 \mathrm{ng} / \mathrm{mL}$. All patients underwent transrectal ultrasonography (TRUS) with biopsies. PSADTZ was estimated by dividing the PSA value by the volume of the transition zone (TZ) obtained. We compared performance measurements for these parameters with those from the PSA itself, PSA density (PSAD) and free PSA/total PSA ratio (F/T PSA). The ability of the method in increasing PSA specificity was demonstrated and compared in univariate and multivariate analyses, and by Receiver Operating Characteristic Curves (ROC).

Results: Of the 68 patients under study, 17 (25\%) were diagnosed with PCa. The TZ volume $(\mathrm{p}=0.001)$ and PSADTZ $(\mathrm{p}=0.001)$ variables presented means that exhibited statistically significant differences. When compared with the area under the curve (AUC), ROC curves obtained by this method revealed that PSADTZ was the strongest predictor for PCa when considering the cut-off point provided by the curve; that is, $0.35 \mathrm{ng} / \mathrm{mL} / \mathrm{cc}$. When PSADTZ was employed, the detection failure would be close to $20 \%$, and less than $45 \%$ of cases would undergo unnecessary biopsies. On the other hand, when F/T PSA was used, the loss would reach almost $40 \%$; however less than $30 \%$ would undergo unnecessary biopsies. Nevertheless, PSADTZ had the only AUC presenting $p<0.05$ in significance when compared with $50 \%$, and was consequently discriminative.

Conclusions: PSADTZ increased PSA specificity in early detection of PCa in males with PSA between 4.0 and $10 \mathrm{ng} / \mathrm{mL}$. However, it was shown to have lower predictive value and lower accuracy than the percentage of free PSA since it presents a higher negative predictive value than all other parameters assessed, and it can be considered clinically useful for reducing unnecessary indications for biopsy.
\end{abstract}

Key words: prostatic neoplasms; diagnosis; prostate-specific antigen; neoplasm staging Int Braz J Urol. 2005; 31: 137-46

\section{INTRODUCTION}

Prostate cancer $(\mathrm{PCa})$ is considered the most frequent neoplasia in men and the second cause of death by cancer in males. Over the past 12 years, the incidence of PCa has increased over $50 \%$ and deaths from the disease risen $40 \%$ (1).

The isolation of the prostate-specific antigen (PSA) in 1979 led to a dramatic change in the diagnosis and management of PCa (2). PSA is the most 
sensitive serum marker in males with prostate disease. Though periurethral glands are known to release PSA into the urine, it is usually accepted that, at least for practical purposes, PSA is produced exclusively by prostate epithelial cells (3).

Therefore, PSA has proven its usefulness as a serum marker for PCa. However, PSA sensitivity and specificity are not yet enough to turn it into the ideal screening test for $\mathrm{PCa}$, since increased levels can also be seen in prostatitis and benign prostate hyperplasia (BPH) (4). The majority of PSA that is released by the prostate into the serum comes from the transition zone and BPH results almost exclusively from the transition zone hyperplasia. Kalish et al. (5) introduced the concept of serum PSA adjusted for the transition zone volume (called PSADTZ) and some studies, like his own, have suggested higher accuracy of this method for early detection of PCa. From all the referenced studies, we can extract a clear impression that their conclusions are not definitive. The introduction of this new concept opens a wide range of new diagnostic possibilities; however, though its usefulness has been statistically demonstrated, it must still be confirmed and validated through other studies that are equally well controlled and methodologically well conducted (reproducible).

In this study, we intend to assess the potential role of PSADTZ in the early diagnosis of PCa in our setting through measurement of early detection from a screening study.

\section{MATERIALS AND METHODS}

This research has been performed with a cross-sectional design in a screening study. The study factor was PSADTZ and other tests for early diagnosis of $\mathrm{PCa}$, and the end-point was the pathological identification of $\mathrm{BPH}$ or $\mathrm{PCa}$.

The sample consisted of 68 patients undergoing screening for early diagnosis of PCa and with indication of transrectal ultrasound (TRUS) with biopsy (altered PSA and/or digital rectal examination). In this research, population, in relation to PSA, only individuals with total PSA values between 4.0 and $10 \mathrm{ng} / \mathrm{mL}$ were included.
The exclusion criteria were a total PSA lower than $4.0 \mathrm{ng} / \mathrm{mL}$ or higher than $10 \mathrm{ng} / \mathrm{mL}$, patient refusal to participate in any assessment procedure, a history of $\mathrm{PCa}$, prostatitis, prostate intraepithelial neoplasia (PIN), urinary tract infection, bladder catheterization or urinary retention and previous prostate surgery.

Variables under study were total prostate volume, transition zone volume, total PSA, free PSA, free-to-total PSA ratio, age, PSADTZ, result of biopsy and digital rectal examination.

All patients from the screening study were assessed for lower urinary tract symptoms by completing the international prostate symptom score, then had their blood collected for determining PSA levels and underwent digital rectal examination.

TRUS with prostate biopsy was indicated for patients who presented changes in their digital rectal examination, which suggested the presence of $\mathrm{PCa}$, and for those with total PSA higher than $4.0 \mathrm{ng} / \mathrm{mL}$. Also, regardless of any adjustments for age, their free PSA was dosed in serum before the TRUS. The serum PSA level was determined by radioimmunoassay method performed on serum collected before digital rectal examination and analyzed with the ImmulitePSA kit (CA, USA).

A digital rectal examination was classified as suspected or non-suspected for neoplasia.

The TRUS measured the total prostate volume and the specific volume of the transition zone (TZ) through a bi-planar 2-probe transductor with 7.5 $\mathrm{MHz}$ in the sagittal probe and $6.5 \mathrm{MHz}$ in the transversal probe, coupled to a model AI 5200 S Envision Acoustic Imaging device (Dornier Co.). Prostate was measured in its transversal and sagittal planes using the formula for estimating the ellipsoid volume (width $\mathrm{x}$ length $\mathrm{x}$ height $\mathrm{x} 0.52$ ). Once the measures were obtained, sextant puncture biopsies were systematically performed using a 16-gauge needle mounted on a Pro-Mag 2.2 automatic pistol.

Biopsy results were generically classified as $\mathrm{PCa}$ or $\mathrm{BPH}$ and/or prostatitis.

The value of PSADTZ was estimated by dividing the serum PSA level value (between 4.0 and $10 \mathrm{ng} / \mathrm{mL}$ ) by the value obtained in an echographic measurement of the $\mathrm{TZ}$ volume and expressed as ng/ $\mathrm{mL} / \mathrm{cc}$. 
As a post-diagnosis approach, individuals who were identified as having PCa were referred to the staging protocol and their therapeutic follow-up was based on the protocol's findings.

\section{Statistical Analysis}

Receiver Operating Characteristic (ROC) curves were used to graphically demonstrate the sensitivity and specificity of the assessed tests (PSA parameters), over a variety of "cut-off" points. The area under the curve (AUC) was calculated using PEPI version 3.0 (Computer Programs for Epidemiologists by J.H. Abramson and P.M. Gahlinger).

Different AUCs were compared as described by Hanley \& McNeil, using the correlation coefficient corrected for AUC as a test for comparing proportions, while the McNemar test uses data that have been previously categorized by their cut-off points.

Wilcoxon-Mann-Whitney U test (WMW) was used for non-parametric analysis of intergroup comparison of data from patients with and without $\mathrm{PCa}$, considering the analysis of means and medians. The relationship between variables was analyzed using Spearman's correlation coefficient.

On the other hand, the linear correlation coefficient (Pearson Product-Moment) was calculated in order to assess the association intensity between total gland volume, TZ volume and PSA.

Finally, we used the multivariate logistic regression analysis model in the "stepwise" system for assessing PSA parameters in relation to its ability to predict PCa by applying systematic selection procedures based on data from the general and negative digital rectal examination groups. The statistical sig- nificance level established for all described tests was $\mathrm{p}<0.05$.

\section{RESULTS}

Of the 68 assessed patients, 17 were diagnosed as having $\mathrm{PCa}$, and $\mathrm{BPH}$ was detected in 51 . The digital rectal examination revealed changes suggestive of malignant neoplasia in 15 patients $(22.1 \%)$ where the presence of PCa was confirmed in only 4 $(26.7 \%)$. Additionally, among patients with normal (unsuspected) digital rectal examination, histology compatible with malignancy was subsequently found in $13(24.5 \%)$ cases. These findings indicate that, in this study group, due to its low sensitivity (23\%), the digital rectal examination was not good as a method for early diagnosis of $\mathrm{PCa}$, despite its good specificity $(78 \%)$ and low positive (29\%) and negative (24\%) predictive value.

When considering their asymmetrical distribution, mean values for parameters in both groups, cancer and benign disease, did not show statistically significant differences for the variables age, PSA, total gland volume and PSAD. On the other hand, the variables TZ volume $(\mathrm{p}=0.001)$ and PSADTZ $(\mathrm{p}=0.001)$ presented means that revealed statistically significant differences in both groups. For estimating the means, the $p$ value considered the Levene's test for equality of variances when applying the $t$ test for independent samples (Table-1).

In relation to the median analysis, the only variables presenting statistical differences were TZ volume $(\mathrm{p}=0.0002)$ and PSADTZ $(\mathrm{p}=0.0012)$ (Table-2).

Table 1 - Association between pathology examination and PSA parameters (mean) in the general group.

\begin{tabular}{lcccc}
\hline & Total & Benign & Cancer & P value \\
\hline PSA & 6.1 & 6.2 & 5.7 & 0.207 \\
Total Volume & 38.3 & 39.9 & 33.8 & 0.153 \\
PSAD & 0.18 & 0.16 & 0.17 & 0.534 \\
TZ Volume & 18.6 & 21.2 & 10.9 & $0.001^{*}$ \\
PSADTZ & 00.48 & 0.36 & 0.62 & $0.001^{*}$ \\
F/T PSA & 00.12 & 0.11 & 0.14 & 0.190 \\
\hline
\end{tabular}

$p<0.05$ Student's " $t$ " test; * significant

$P S A D=P S A$ density $; T Z=$ transition zone $; P S A D T Z=P S A$ density in the transition $z o n e ; F / T=$ free/total 
Table 2 - Association between pathology examination and PSA parameters (medians) in the general group.

\begin{tabular}{lcccc}
\hline & Total & Benign & Cancer & P value \\
\hline PSA & 5.6 & 5.6 & 5.6 & 0.565 \\
Total Volume & 35.7 & 36.5 & 35.2 & 0.218 \\
PSAD & 0.16 & 0.16 & 0.18 & 0.470 \\
TZ Volume & 16.8 & 18.5 & 9.5 & $0.001^{*}$ \\
PSADTZ & 0.36 & 0.31 & 0.7 & $0.001^{*}$ \\
F/T PSA & 0.11 & 0.11 & 0.13 & 0.145 \\
\hline
\end{tabular}

$p<0.05$ Mann-Whitney " $U$ " test. * significant

$P S A D=P S A$ density $; T Z=$ transition zone $; P S A D T Z=P S A$ density in the transition zone; $F / T=$ free/total

Bivariate analysis of correlations between total prostate volume and TZ volume using the scatter plot presented a regression straight line (Figure1) as demonstrated by the plot's points, suggesting a strong correlation between them $(\mathrm{r}=0.825, \mathrm{p}=$ $0.0001)$. On the other hand, when analyzing PSA and other volumes, the correlation coefficient was very low both at the crossing of PSA and total volume $(r=$ $0.05, \mathrm{p}=0.664)$ and at the crossing of PSA and TZ volume $(\mathrm{r}=0.01, \mathrm{p}=0.928)$.

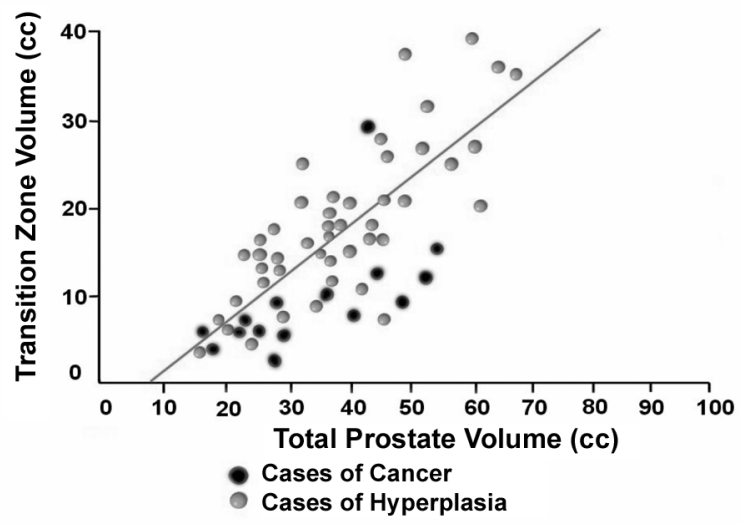

Figure 1 - Scatter graph with regression straight line showing the correlation between total prostate volume ( $X$ axis) and transition zone volume ( $Y$ axis).
Data on each parameter shown in the Tables demonstrating performance measurements were obtained from the best cut-off value for each parameter (which, in turn, was determined by the ROC curve), considering both the general and the negative digital rectal examination groups (Table-3). In relation to absolute values in the negative digital rectal examination group, the AUC of PSADTZ presented higher absolute values than did F/T PSA, in addition to being more significant (considering the significance compared with $50 \%$ ). Its specificity, positive predictive value (PPV) and accuracy are surpassed by the performance f F/T PSA; however, its sensitivity and its negative predictive value (NPV) are clearly superior.

When compared, ROC curves obtained for each parameter from their specific cut-off values revealed that PSADTZ was the strongest predictor of PCa when considering the cut-off point in the upper left area of the curve - that is $0.35 \mathrm{ng} / \mathrm{mL} / \mathrm{cc}$ (Figure2). It means that when considering the best cut-off point for PSAD $(0.15 \mathrm{ng} / \mathrm{mL} / \mathrm{cc})$, in the group with negative digital rectal examination approximately $40 \%$ of PCa cases would be missed (since sensitivity was approximately $60 \%$ ) and about $60 \%$ would undergo unnecessary biopsies (since specificity was approximately 40\%). Using the PSADTZ parameter, the missing rate would be close to $20 \%$ and less than $45 \%$ of patients would undergo unnecessary biopsies.

The "box plot" percentile graphs demonstrated central trend values for PSADTZ, whose me- 
Table 3 - Performance measurements for PSA parameters in the general $(G)$ and negative digital rectal examination (DRE) groups.

\begin{tabular}{lcccccc}
\hline Parameters & $\begin{array}{c}\text { Cut-off } \\
\text { Point }\end{array}$ & Specificity & Sensitivity & $\begin{array}{c}\text { Positive Predictive } \\
\text { Value }\end{array}$ & $\begin{array}{c}\text { Negative Predictive } \\
\text { Value }\end{array}$ & Accuracy \\
\hline PSA (G) & 5 & 35.3 & 70.6 & 26.6 & 78.2 & 44.1 \\
PSA (DRE) & 5 & 37.5 & 69.2 & 26.4 & 78.9 & 45.2 \\
PSA (G) & 0.15 & 45.1 & 94.1 & 27.5 & 90.0 & 36.7 \\
PSA (DRE) & 0.15 & 42.5 & 61.5 & 25.8 & 77.2 & 47.1 \\
Free PSA (G) & 0.15 & 78.2 & 50.0 & 46.1 & 80.6 & 70.4 \\
Free PSA (DRE) & 0.15 & 72.0 & 62.5 & 41.7 & 85.7 & 69.7 \\
PSADTZ (G) & 0.35 & 56.9 & 82.4 & 38.8 & 90.6 & 63.2 \\
PSADTZ (DRE) & 0.35 & 55.0 & 76.9 & 35.7 & 88.0 & 60.3 \\
\hline
\end{tabular}

PSADTZ = PSA density in the transition zone $F / T=$ free/total

dian was $0.62 \mathrm{ng} / \mathrm{mL} / \mathrm{cc}$ for PCa and $0.28 \mathrm{ng} / \mathrm{mL} / \mathrm{cc}$ for BPH. The cut-off value for PSADTZ was 0.36 $\mathrm{ng} / \mathrm{mL} / \mathrm{cc}$, which corresponded to values between the $75^{\text {th }}$ percentile for cancer $(75 \%$ of cases of PCa were above this value) and the $25^{\text {th }}$ percentile for benign disease ( $75 \%$ of cases of BPH were below this value). When considering the values obtained by the same analysis, we verified a statistically significant difference between PSADTZ values for both groups $(\mathrm{p}=$ 0.0012), Figure-3.

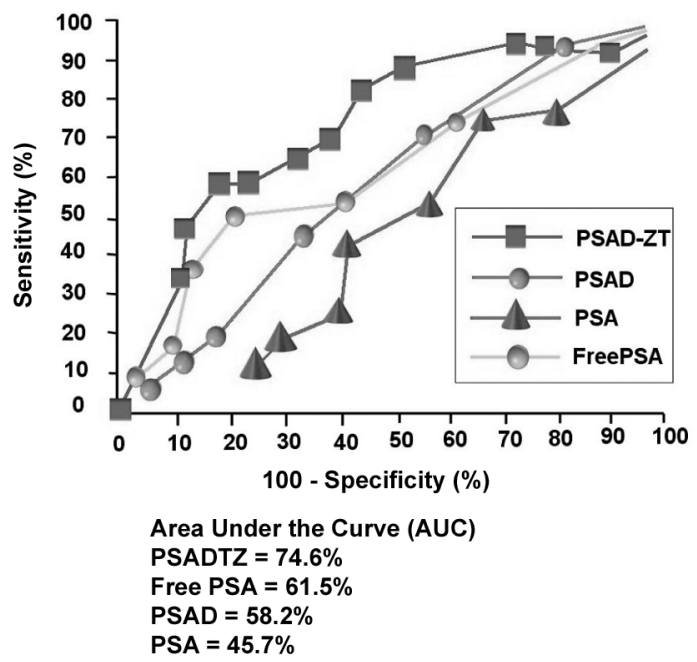

Considering the ROC curves for each parameter in the parameter analysis, PSA presented a smaller area $(\mathrm{AUC}=45.7 \%)$ and low specificity $(35 \%)$, while PSADTZ showed a larger area $(\mathrm{AUC}=74.6 \%)$ and higher specificity $(56.9 \%)$ and was the only parameter to present significance compared with $50 \%$ at significant levels; that is, it was discriminative. At its best cut-off point $(0.35 \mathrm{ng} / \mathrm{mL} / \mathrm{cc})$ whose sensitivity reached $82.4 \%$, there would be a more accentuated reduction in the number of unnecessary biopsies (over

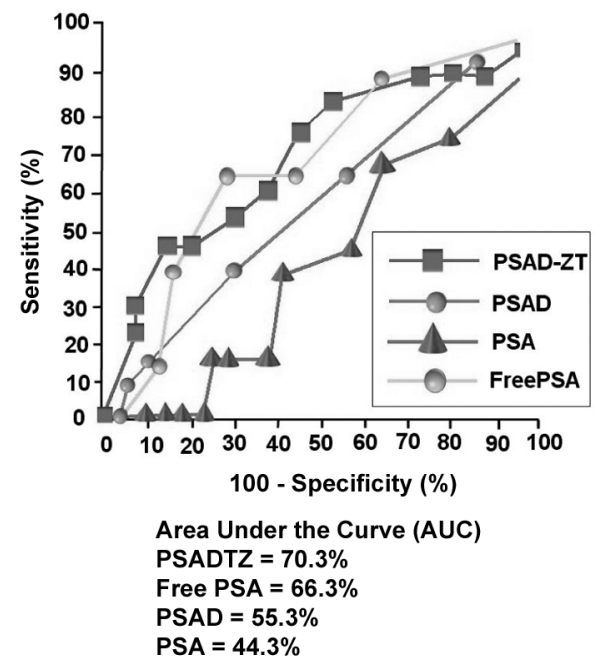

Figure 2 - Comparison of ROC curves with specification of AUCs in the general (left) and negative digital rectal examination (right) groups. $P S A D-Z T=P S A$ density in the transition zone. 


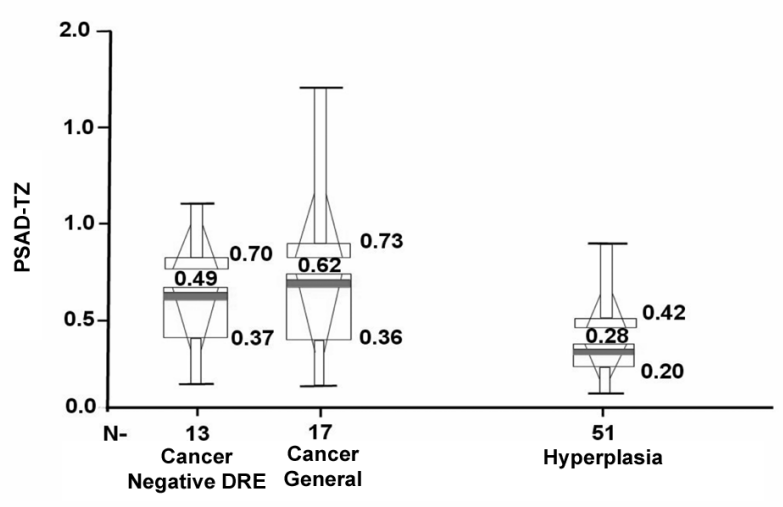

Figure 3 - "Box Plot" graphs showing the distribution of mean and median PSADTZ values for cancer $(n=17)$ and hyperplasia $(n=51)$ in the general and negative digital rectal examination $(n=13)$ groups. $P S A D-Z T=P S A$ density in the transition zone.

$55 \%$ ) in comparison with other diagnostic tests (PSA parameters), Table-4.

In the comparative analysis of the respective AUCs, using the Hanley-McNeil method, statistically significant differences were observed between the AUC of the PSADTZ parameter and those of the PSA and PSAD parameters (Table-5). The area under the curve of the parameter F/T PSA, however, was not statistically different from the PSADTZ, thus suggesting a similar overall performance for both tests in increasing PSA specificity.
Results obtained with the multiple logistic regression analysis model for prediction of $\mathrm{PCa}$, which applied the "stepwise" selection procedure based on the 4 variables, excluded non-significant variables - that is PSA and PSAD - demonstrating that PSADTZ and free-to-total PSA ratio were the strongest and most significant predictors of PCa. When considering values obtained at the best cutoff points in the original ROC curves, the odds ratio was 9.07 (with $\mathrm{p}=0.032$ ) for PSADTZ and 7.45 (with $p=0.027$ ) for free-to-total ratio. When the assessment was performed using cut-off points with highest specificity of the ROC curve, odds ratio was $13.71(\mathrm{p}=0.005)$ and $6.33(\mathrm{p}=0.069)$ respectively, showing statistical superiority of PSADTZ in predicting PCa (Table-6).

\section{COMMENTS}

Studies have indicated that $2 / 3$ of patients undergoing biopsy based exclusively on the finding of an intermediary PSA value show no histological evidence of $\mathrm{PCa}$ (6). In this respect, several new concepts have been introduced regarding PSA, all of them intended to optimize the clinical usefulness of PSA by increasing its sensitivity and specificity and, thus, trying to reduce the numbers of unnecessary biop-

Table 4 - Analytical and descriptive results from performance measures of parameters in the general $(G)$ and negative digital rectal examination (DRE) groups.

\begin{tabular}{lcccccccc}
\hline Parameters & $\begin{array}{l}\text { PSA } \\
\text { (G) }\end{array}$ & $\begin{array}{c}\text { PSA } \\
\text { (DRE) }\end{array}$ & $\begin{array}{c}\text { PSAD } \\
(\mathbf{G})\end{array}$ & $\begin{array}{c}\text { PSAD } \\
(\mathbf{D R E})\end{array}$ & $\begin{array}{c}\text { F/T PSA } \\
(\mathbf{G})\end{array}$ & $\begin{array}{c}\text { F/T PSA } \\
\text { (DRE) }\end{array}$ & $\begin{array}{c}\text { PSADTZ } \\
\text { (G) }\end{array}$ & $\begin{array}{c}\text { PSADTZ } \\
\text { (DRE) }\end{array}$ \\
\hline $\begin{array}{l}\text { Comparison Significance } \\
\quad \text { in AUC *** }\end{array}$ & 0.71 & 0.75 & 0.13 & 0.28 & 0.12 & 0.06 & $0.001^{*}$ & $0.008^{*}$ \\
AUC & & & & & & & & \\
Best Cut-off Point & 45.7 & 44.3 & 58.2 & 55.3 & 61.5 & 66.3 & 74.6 & 70.3 \\
Sensitivity & 5 & 5 & 0.15 & 0.15 & 0.15 & 0.15 & 0.35 & 0.35 \\
Specificity & 70.6 & 69.2 & 94.1 & 61.5 & 50.0 & 62.5 & 82.4 & 76.9 \\
Positive Predictive Value & 35.3 & 37.5 & 45.1 & 42.5 & 78.2 & 72.0 & 56.9 & 55.0 \\
Negative Predictive Value & 78.6 & 26.5 & 27.5 & 25.8 & 46.1 & 41.7 & 38.8 & 35.7 \\
Accuracy & 74.1 & 45.2 & 90.0 & 77.2 & 80.6 & 85.7 & 90.6 & 88.0 \\
\hline
\end{tabular}

* significant value, ** significance compared with 50\% in AUC considering $p<0.05, A U C=$ area under the curve $P S A D=P S A$ density; $T Z=$ transition zone $; P S A D T Z=P S A$ density in the transition zone $; F / T=$ free/total 
Table 5 - Comparative analysis of areas under the curves (AUCs) of PSA density in the transition zone with areas AUCs of the other parameters in the general and negative digital rectal examination groups.

\begin{tabular}{lcccccl}
\hline PSA Parameters & AUCs of PSADTZ & $\begin{array}{c}\text { AUCs of other } \\
\text { parameters }\end{array}$ & r value & $\begin{array}{c}\text { Means of } \\
\text { Compared AUCs }\end{array}$ & Z value & P value \\
\hline PSA (G) & 74.6 & 45.7 & 0.3673 & 60.15 & 3.459 & $0.001^{*}$ \\
PSA (DRE) & 70.3 & 44.3 & 0.3028 & 57.3 & 2.559 & $0.01^{*}$ \\
PSAD (G) & 74.6 & 58.2 & 0.7950 & 66.4 & 3.321 & $0.001^{*}$ \\
PSAD (DRE) & 70.3 & 55.3 & 0.6940 & 62.8 & 2.102 & $0.036^{*}$ \\
F/T PSA (G) & 74.6 & 61.5 & -0.107 & 68.05 & 1.124 & 0.261 \\
F/T PSA (DRE) & 70.3 & 66.3 & -0.249 & 68.30 & 0.328 & 0.743 \\
\hline
\end{tabular}

$* p<0.05, r=$ Pearson's correlation coefficient, $Z=$ Coefficient of Hanley-McNeil table

$P S A D=P S A$ density $; Z=$ transition zone $; P S A D T Z=P S A$ density in the transition $z o n e ; F / T=$ free/total

sies in men with benign disease. These new concepts include PSA density, PSA velocity, PSA adjusted for age, and determination of molecular forms of PSA (free versus conjugated to proteins) $(7,8)$.

For this purpose, the concept of PSADTZ has been recently suggested as a valuable approach. When comparing the results from the assessment of mean and median values for the parameters obtained in our study with those demonstrated by the majority of reviewed authors, we found an important similarity in the significant differences obtained in the 2 subgroups ( $\mathrm{PCa}$ and $\mathrm{BPH}$ ) for the variables $\mathrm{TZ}$ volume and PSADTZ (6-8).

On bivariate analysis of correlations, results observed in the literature have presented correlation coefficients between the variables total volume and TZ volume that, when compared, confirm those obtained in our study (7,9-11). Comparative analyses of the areas under ROC curves of several parameters demonstrate that higher indexes are observed by the PSADTZ parameter, both in the total group and in the segment's negative digital rectal examination, volume $<30 \mathrm{cc}$ and volume $\geq 30 \mathrm{cc}$. These results are comparable in the total group to those found by other authors $(6,7,9-13)$. However, in a deviation from the study by Maeda et al. (7) who detected a higher index in the group with negative digital rectal examination, the present project did not attain such an outcome. On the other hand, Lin et al. (15), Akduman et al. (16), Gohji et al. (17) and Rietbergen et al. (18) did not find significant statistical differences between the AUCs of PSAD and PSADTZ, even if they used different sampling criteria and statistical methods in relation to our study.

When using the method described by Hanley and $\mathrm{McNeil}$ for comparison between the AUC parameters estimated both for the total group and for the group with negative digital rectal examination, we

Table 6 - Multivariate analysis by logistic regression considering the best cut-off point $(C P)$ and sensitivity $(S)$ of 90 $95 \%$.

\begin{tabular}{lccccr}
\hline Variable & Estimate $*$ & Standard Error & Wald $* *$ & Significance $* * *$ & Odds Ratio \\
\hline PSADTZ (CP) & 2.2055 & 1.0283 & 4.60 & 0.032 & 9.074 \\
PSADTZ (S) & 2.6188 & 0.9491 & 7.61 & 0.005 & 13.718 \\
F/T PSA (CP) & 2.0084 & 0.9118 & 4.85 & 0.027 & 1.248 \\
F/T PSA (S) & 1.8457 & 1.0169 & 3.29 & 0.069 & 0.863 \\
\hline
\end{tabular}

* test's coefficient, ** test's $p$, *** p value

$P S A D=P S A$ density $; T Z=$ transition zone $; P S A D T Z=P S A$ density in the transition $z o n e ; F / T=$ free/total 
observed that they showed significant differences, especially when comparing PSADTZ with the PSA and PSAD parameters. Theses differences have been demonstrated by other studies as well, thus revealing the high predictive power of this diagnostic test $(6,7,10,12)$.

On the other hand, when PSADTZ was compared with the F/T PSA parameter, no statistically significant difference was found in either group $(\mathrm{p}=0.261$ and $p=0.743$, respectively). The same result has been obtained by the majority of previous studies observing the absolute value of AUC for PSADTZ $(6,7,9,11,13,14)$. Searching for a cut-off limit for all parameters that maintained the sensitivity for cancer detection around minimal limits of 90 to $95 \%$, values were defined that, in addition to being close to those evidenced in the literature (except for F/T PSA), presented very similar corresponding specificity as well with no exceptions. At $0.3 \mathrm{ng} / \mathrm{mL} / \mathrm{cc}$, PSADTZ reached $47 \%$ of specificity, the same one obtained by the cutoff value of 0.25 in the study by Djavan et al. (6).

Finally, by applying the "stepwise" selection procedure which has been performed by a few authors, our results were in agreement with those demonstrated by Djavan et al. $(6,12)$ in 2 of their studies ( $n=559$ and $n=1051$ respectively) where the same exclusions for the PSA and PSAD parameters were performed due to their low predictive value in both studies, thus revealing the F/T PSA and PSADTZ parameters as the strongest and highly predictive for PCa. This result was also similar to the one presented by Moon et al. (9).

Among limitations observed in our analysis is the sample " $n$ " itself, which, despite being regarded as reduced and consequently presenting a wide confidence interval, was surprisingly able to provide statistical results that were comparable to larger series. Analyses related to the predictive ability for extraprostatic disease were not performed as well, despite being the object of study in some of the reviewed references (19).

The need for cost-effectiveness studies is evident as the main components of this diagnostic test involve the performance of 2 procedures, which, in turn, have their specific costs - even if we can infer a decreased cost when considering savings due to fewer indications of TRUS with biopsies, its own associated costs, and risks that are certainly more relevant. Another fact to be considered, which is still unedited, is the proposal for using the method presented here in the routine for performance of transrectal biopsies in patients with indication who, in case of a benign diagnosis even in the presence of suggestive changes (PSA and/or digital recital examination) could benefit from another parameter for increasing the specificity of PSA when altered values are maintained. When considering the possibility in the near future that these patients undergo a new invasive procedure that is not exempt from risks, we must remember the recent studies published by Okegawa et al. (20) and Singh et al. (21), showing that PSADTZ, as well as advanced age and presence of high grade PIN, is one of the strongest predictors for $\mathrm{PCa}$ in subsequent biopsies following the initial biopsy.

\section{CONCLUSION}

The PSA parameter known as PSADTZ has shown itself to possess a statistical power for increasing the specificity, positive predictive value, negative predictive value and accuracy, which, in addition to being similar to performance measures verified in the comparative analysis with F/T PSA, were superior to the latter in some analyses. The best cut-off point established by our study was $0.35 \mathrm{ng} / \mathrm{mL} / \mathrm{cc}$.

\section{REFERENCES}

1. Parker SL, Tong T, Bolden S, Wingo PA: Cancer statistics, 1996. CA Cancer J Clin. 1996; 46: 5-27.

2. Schellhammer PF, Wright GL: Biomolecular and clinical characteristics of PSA and other candidate prostate tumor markers. Urol Clin North Am. 1993; 4: 597 606.

3. Noldus J, Stamey TA: Limitations of serum prostate specific antigen in predicting peripheral and transition zone cancer volumes as measured by correlation coefficients. J Urol. 1996; 155: 232-7.

4. Partin AW, Oesterling JE: The clinical usefulness of prostate specific antigen: update 1994. J Urol. 1994; 152: 1358-64.

5. Kalish J, Cooner WH, Graham SD Jr.: Serum PSA adjusted for volume of transition zone (PSAT) is more 
accurate than PSA adjusted for total gland volume (PSAD) in detecting adenocarcinoma of the prostate. Urology. 1994; 43: 601-7.

6. Djavan B, Zlotta AR, Byttebier G, Shariat S, Omar M, Schulman CC, et al.: Prostate specific antigen density of the transition zone for early detection of prostate cancer. J Urol. 1998; 160: 411-8; discussion 418-9.

7. Maeda H, Arai Y, Ishitoya S, Okubo K, Aoki Y, Okada T: Prostate specific antigen adjusted for the transition zone volume as an indicator of prostate cancer. J Urol. 1997; 158: 2193-6.

8. Catalona WJ, Smith DS, Wolfert RL, Wang TJ, Rittenhouse HG, Ratliff TL, et al.: Evaluation of percentage of free serum prostate-specific antigen to improve specificity of prostate cancer screening. JAMA. 1995; 274: 1214-20.

9. Moon DG, Cheon J, Kim JJ, Yoon DK, Koh SK: Prostate-specific antigen adjusted for the transition zone volume versus free-to-total prostate-specific antigen ratio in predicting prostate cancer. Int J Urol. 1999; 6: 455-62.

10. Kurita Y, Terada H, Masuda H, Suzuki K, Fujita K: Prostate specific antigen (PSA) value adjusted for transition zone volume and free PSA (gammaseminoprotein)/PSA ratio in the diagnosis of prostate cancer in patients with intermediate PSA levels. Br J Urol. 1998; 82: 224-30.

11. Michielsen DP, De Boe VR, Braeckman JG, Keuppens FI: Specificity and accuracy of TRUS-measured PSAdensity and transition zone-PSA in the diagnosis of prostate cancer. Eur J Ultrasound. 1998; 8: 125-8.

12. Djavan B, Zlotta A, Remzi M, Ghawidel K, Basharkhah A, Schulman CC, et al.: Optimal predictors of prostate cancer on repeat prostate biopsy: a prospective study of 1,051 men. J Urol. 2000; 163: 1144-1148; discussion 1148-49.

13. Kikuchi E, Nakashima J, Ishibashi M, Ohigashi T, Asakura H, Tachibana M, et al.: Prostate specific antigen adjusted for transition zone volume: the most pow- erful method for detecting prostate carcinoma. Cancer. 2000; 8: 842-9.

14. Roumeguere T, Zlotta AR, Djavan BR, Marberger M, Schulman CC: PSA level of the transitional zone: a new marker especially reliable for the detection of prostatic cancer. Acta Urol Belg. 1997; 65: 5-9.

15. Lin DW, Gold MH, Ransom S, Ellis WJ, Brawer MK: Transition zone prostate specific antigen density: lack of use in prediction of prostatic carcinoma. J Urol. 1998; 160: 77-81; discussion 81-2.

16. Akduman B, Alkibay T, Tuncel A, Bozkirli I: The value of percent free prostate specific antigen, prostate specific antigen density of the whole prostate and of the transition zone in Turkish men. Can J Urol. 2000; 7: 1104-9.

17. Gohji K, Nomi M, Egawa S, Morisue K, Takenaka A, Okamoto M, et al.: Detection of prostate carcinoma using prostate specific antigen, density and the density of the transition zone in Japanese men with intermediate serum prostate specific antigen concentrations. Cancer. 1997; 79: 1969-76.

18. Rietbergen JB, Kranse R, Hoedemaeker RF, Kruger AE, Bangma CH, Kirkels WJ, et al.: Comparison of prostate-specific antigen corrected for total prostate volume and transition zone volume in a populationbased screening study. Urology. 1998; 52: 237-46.

19. Horiguchi A, Nakashima J, Horiguchi Y, Nakagawa K, Oya M, Ohigashi T, et al.: Prediction of extraprostatic cancer by prostate specific antigen density, endorectal MRI, and biopsy Gleason score in clinically localized prostate cancer. Prostate. 2003; 56: 23-9.

20. Okegawa T, Kinjo M, Ohta M, Miura I, Horie S, Nutahara K, et al.: Predictors of prostate cancer on repeat prostatic biopsy in men with serum total prostate-specific antigen between 4.1 and $10 \mathrm{ng} / \mathrm{mL}$. Int J Urol. 2003; 10: 201-6.

21. Singh H, Canto EI, Shariat SF, Kadmon D, Miles BJ, Wheeler TM, et al.: Predictors of prostate cancer after initial negative systematic 12 core biopsy. J Urol. 2004; 171: 1850-4.
Received: September 20, 2004 Accepted after revision: March 17, 2005
Correspondence address:

Dr. Marcos Dias Ferreira

Rua Ladislau Neto, 474 / casa 01, Bairro Ipanema

Porto Alegre, RS, 91760-070, Brazil

Fax: + $55513249-9653$ 


\section{EDITORIAL COMMENT}

In this study, the authors used ultrasound to determine the volume of the transition zone where $\mathrm{BPH}$ would be expected to occur and where cancer is less likely to occur. When the PSA is factored by the volume of the transition zone, they found that the PSA density in transition zone was more predictive of prostate cancer than either serum total PSA or PSA density as routinely determined in patients with a PSA of 4 to $10 \mathrm{ng} \%$. The percentage of free PSA and transition zone PSA density enhance the specificity of PSA when determining which patients should undergo repeat biopsy. Overall, $25 \%$ of the patients were diagnosed with prostate cancer. The high cancer rate can be explained by the fact that patients included in this study were referred for early diagnosis and not for screening purposes. Accuracy of transition zone volume measurement is ultrasonographer-dependent, which may influence the reproducibility of the pro- cedure. Of some concern may be the fact that the predictive power of the PSA transition zone is significantly affected by prostate size in prostates weighing less than $30 \mathrm{~g}$ in which the transition zone is not markedly enlarged and is therefore more difficult to measure. The usefulness of the PSA transition zone density is significantly diminished and less accurate than free-to-total PSA (1). Finally, unlike PSA parameters, PSA density in the transition zone requires the use of transrectal ultrasound, escalating the costs of the test.

\section{REFERENCE}

1. Djavan B, Zlotta A, Kratzik C, Remzi M, Seitz C, Schulman CC, et al.: PSA, PSA density, PSA density of transition zone, free/total PSA ratio, and PSA velocity for early detection of prostate cancer in men with serum PSA 2.5 to $4.0 \mathrm{ng} / \mathrm{mL}$. Urology. 1999; 54: 517-22.

Dr. Valdemar Ortiz

Division of Urology

Federal University of Sao Paulo, UNIFESP

São Paulo, SP, Brazil 